\title{
A Comparison Performance Analysis of QoS WLANs: Approaches with Enhanced Features
}

\author{
Ioannis Papapanagiotou, ${ }^{1}$ Georgios S. Paschos, ${ }^{2}$ and Michael Devetsikiotis ${ }^{1}$ \\ ${ }^{1}$ Department of Electrical and Computer Engineering, North Carolina State University, P.O. 27695-7911, Raleigh, NC 27695, USA \\ ${ }^{2}$ National Research Institute of Finland VTT, 02150 Espoo, Finland
}

Received 30 May 2007; Accepted 13 July 2007

Recommended by Stavros Kotsopoulos

The main contribution of this work is to compare and enhance known methods for performance analysis of the IEEE 802.11e MAC layer, such as the use of Markov chains, queuing theory, and probabilistic analysis. It is the first paper that bases its outputs upon comparison of metrics such as complexity, flexibility, and accuracy, leading to the novel use of a metamodeling comparison. For the analysis, complexity theory and the $L$-square distance method for accuracy are used. In addition, the proposed analyses carry by themselves scientific interest, because they are extended enhancements with the latest EDCA parameters. A form of the PMF of the MAC delay and first-order moments are found using the PGF complex frequency domain function. The analyses incorporate a Gaussian erroneous channel in order to reflect the real conditions of the MAC layer.

Copyright (c) 2007 Ioannis Papapanagiotou et al. This is an open access article distributed under the Creative Commons Attribution License, which permits unrestricted use, distribution, and reproduction in any medium, provided the original work is properly cited.

\section{INTRODUCTION}

The wide deployment of WLANs has set an increased pace for extensive scientific studies of the IEEE 802.11 standard [1]. In addition, heterogeneous multimedia applications require advanced editing over the standard, so as to accomplish specific QoS characteristics [2]. Accomplishing such QoS features will make capable transmission of video and voice over new 3G-WLANs [3].

The core 802.11e standard proposes a new hybrid coordination function (HCF), which has the HCF controlled channel access (HCCA) and the enhanced distributed coordination access (EDCA) mechanisms, capable of offering access according to specific QoS features. A typical literature search demonstrates a number of performance analyses for the legacy IEEE 802.11 and 802.11e [4-7] or similar enhancements [8], which incorporate discrete time Markov chains (DTMC). Other models, such as [9-11], use alternative methods of analysis.

A trend has developed, since [4] first presented his case study, to improve and provide more accurate performance values. Due to the subject maturity, the space left for new models that could prompt scientific interest is small. Our novel approach is to propose amendments over these known analytical methods, find their accurate values, and open a new field of performance comparison. It is straightforward that, since new protocols tend to be analyzed by either DTMCs, queuing theory, or general probabilistic methods, the results of the proposed methods can be used to find the best method of analyzing forthcoming or known standards.

We have used three known models $[5,10,11]$, which depict the three main methods of analysis, and they are extended according to QoS features proposed in the dot1le standard and error-prone channel. The first model uses DTMC analysis, which takes into account the state of the previous slot. The second one is based on elementary conditional probability arguments, and finally, in the last model, queuing theory and Little's theorem are used to analyze the standard. Accurate values of delay and a way for calculating the PMF of the MAC delay are also given. The proposed analysis alterations carry by themselves scientific interest, and could be studied separately.

Except from some already investigated features of the dot11e, additional ones have been added. These performance enhancements are summarized in the following.

(i) In our models, the effect of different retransmission limits among the access categories is implemented.

(ii) Freezing of backoff counters is taken into account. 
(iii) A more accurate equation of saturation throughput is provided in correlation with a way of incorporating the AIFS differentiation phenomenon among the access categories.

(iv) It was also noted that higher ACs monopolized relatively quickly the channel, especially when the type of multimedia traffic was bursty. After revision of D4.0 of the IEEE 802.11e standard [2], the standard defined that after a successful transmission, the AC should get back to backoff, and contest again for the channel in the next time slot. This means that the state 0 of the DTMC cannot be chosen after a successful transmission a feature that older models have omitted orpartly mentioned [12], but not analyzed.

(v) The proposed models include Gaussian erroneous channel for EDCA. Although some works exist, for example, $[13,14]$, they tend to analyze such problems by implementing the bit error rate (BER) probability in the busy probability, which means that an error can make the channel busy. Since the MAC layer in the backoff level does not see errors and inner codes in the sublayers provide a specific coding gain, the erroneous channel implementation should be made in the performance analysis.

(vi) We analyze in the same environment the effect of Block-ACK and the efficiency of the new IEEE 802.11n introducing much higher transmission rates.

(vii) Most of the aforementioned analyses tend to give results for the basic transfer rate, in which analytical features such as the dual effect of the RTS/CTS in throughput are not shown. In our models, these are corrected while adding new features and giving the exact solution with RTS/CTS, basic access mode in $11 \mathrm{Mbps}$, and multiples of $24 \mathrm{Mbps}$ transfer rate.

(viii) $Z$-transform is also used for the PGF of the MAC delay, and first-order moments are found through its first and second derivatives.

The proposed analysis alterations carry themselves scientific interest, and could be studied separately. Additionally, a metamodeling analysis is added, by processing the mathematical performance and simulation outcomes. Thus results are given in terms of

(i) complexity, based on big-O notation;

(ii) accuracy, based on $L$-square distance;

(iii) depiction of the states of the MAC protocol, nonsaturation easiness of implementation, and flexibility.

The simulation results are based on the HCCA model included in the last version of OPNET modeler 12. The proposed models require advanced knowledge of $[2,4,10]$, since formulas and other proved explanations are taken as prerequisites. The paper is organized as follows. In Section 2, we provide numerical analysis of the transmission probability $\left(\tau_{i}\right)$ and mean backoff duration $\left(E[B D]_{i}\right)$ of each of the three models. In Section 3, taking into account the values of throughput and delay the transmission rates are extracted, with various conditions of the channel and features enabled or disabled. A third subsection is also given for the anal-
TABLE 1: 802.11e EDCA standard parameters.

\begin{tabular}{lcccc}
\hline & AC0 & AC1 & AC2 & AC3 \\
\hline Type of service & VoIP & Video & Best effort & Backround \\
CWmin[i] & 7 & 15 & 31 & 31 \\
CWmax[i] & 15 & 31 & 1023 & 1023 \\
AIFS[i] & SIFS +2 & SIFS +2 & SIFS +3 & SIFS +7 \\
\hline
\end{tabular}

ysis of the Block-ACK feature. In Section 4, validation, results, and comparison analysis and evaluation are provided. In Section 5 a conclusive discussion is made upon advantage and disadvantage of each one.

\section{NUMERICAL ANALYSIS OF THE MODELS}

In [2], quality of service succeeded via using four access categories (ACs) with different transmission parameters each. The standard uses different values of $\operatorname{AIFS}[i], C W \min _{i}$, $C W \max _{i}$ and backoff persistent factor $\left(\pi f_{i}\right), i=\{0,1,2,3\}$. Finally, transmission opportunity (TxOP) is the maximum amount of bytes that a station is allowed to transmit consecutively before it releases the channel. In this paper, all ACs are considered to send packets with equal number of bytes below TxOP limit and therefore TxOP is not studied. We define $W_{i, 0}=C W_{i, \min }+1$, where $W_{i, j}$ is the contention window size and $j$ is the backoff stage. $m_{i}$ is defined as the retry limit, after which the contention window remains the same for a number of retransmissions. When the backoff exponential algorithm reaches $L_{i}$ (long retry limit) times of retransmission and there is a collision, the packet is dropped. In the legacy 802.11 [1], persistent factor $\left(\pi f_{i}\right)$ has the value of 2 , which means that after every collision, the backoff contention window doubles its value. In [2], persistent factor can have different values according to each access category

$$
W_{i, j}= \begin{cases}\left\lfloor\left(\pi f_{i}\right)^{j} W_{i, 0}\right\rfloor, & j=0,1, \ldots, m_{i}, \\ \left\lfloor\left(\pi f_{i}\right)^{m_{i}} W_{i, 0}\right\rfloor, & j=m_{i}+1, \ldots, L_{i}\end{cases}
$$

where $L \cdot\rceil$ is the closest integer function. In Table 1 , a summary of the EDCA is presented.

Before defining the mathematical analysis, the following assumptions have been made regarding all models. The number of stations $N_{i}$ is finite and equal for all ACs and contends only in a single-hop network. There is a constant packet generator and the network is saturated, which means that there is always a packet ready to transmit in each terminal. The channel is erroneous, with uniform distributed errors, and there are no hidden terminal, capture effects, and link-adaptation mechanisms. Finally, as in all existing models, the transmit probability is considered to be independent per station.

\subsection{Markov chain model (model 1)}

A three-dimensional DTMC is proposed, which presents the effect of contending terminals on the channel access probability of each access class (AC), and is described by the stationary probabilities $b_{i, w, j, k}$. The parameter $i=\{0,1,2,3\}$ 


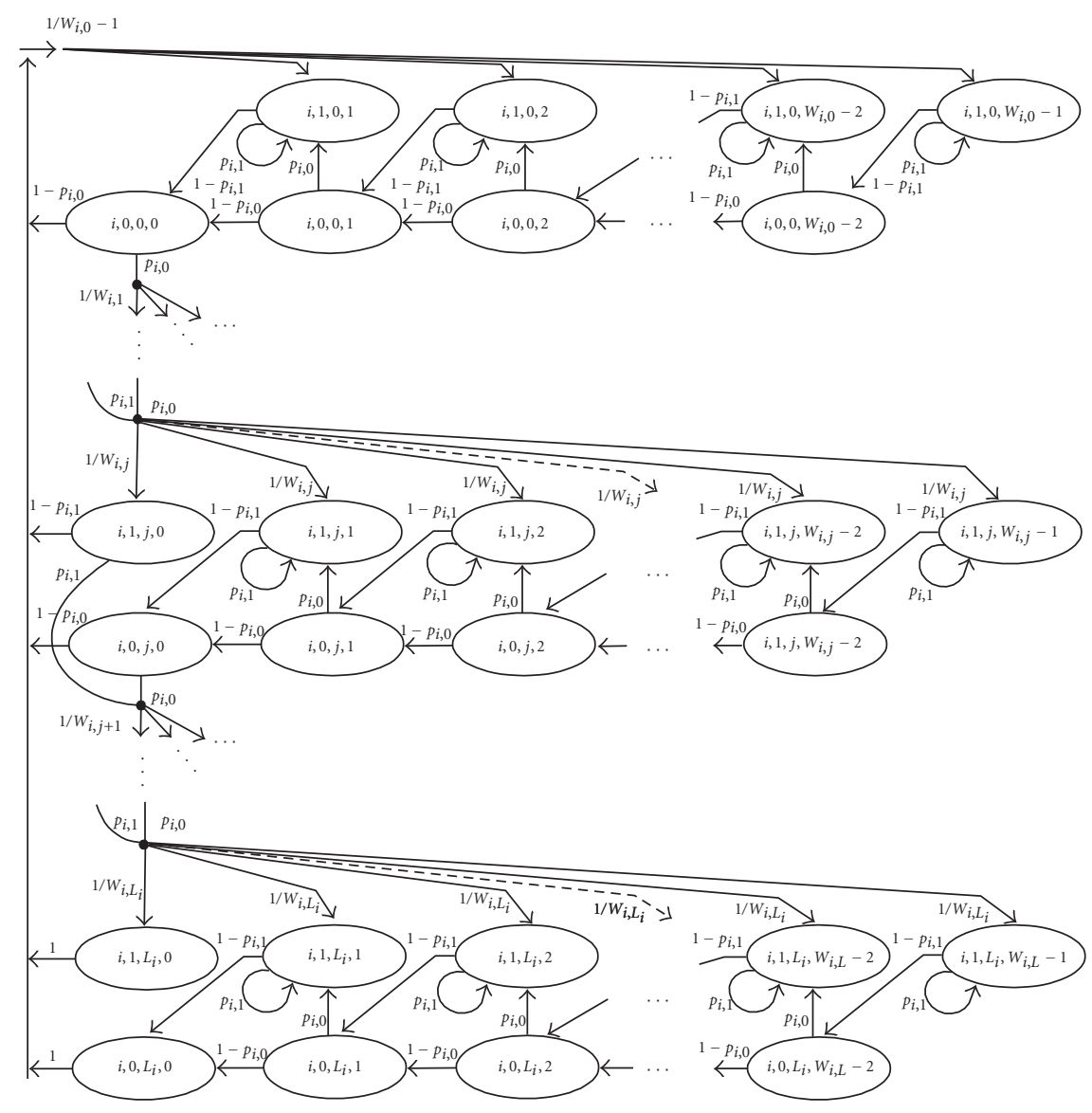

Figure 1: Analytical Markov chain for each access category AC[i].

describes the four access categories, which differentiate the access method according to the dot11e standard.

The first dimension, $w$, represents the condition of the previous slot, where 1 is for the busy channel and 0 for the idle channel. Similar to [5], a division is needed since special cases exist according to the state of the previous slot. If it was idle, all access categories of all stations may access the channel if their backoff counter is decremented to zero.

On the other hand, if the previous slot was busy, another division must take place. A busy slot can occur if there is a collision or a transmission of another station. In the first case, the stations that did not participate in the collision have frozen their backoff counter and will not be able to transmit. Instead, the stations that collided can transmit in the next slot if they choose a new backoff value equal to 0 . In the second case, when there is a successful transmission, none of the stations can transmit in the next time slot. This happens specifically for the standard IEEE 802.11e and not for the legacy IEEE 802.11. The latest defines that after a successful transmission, the contention window starts from 1 and not from 0 . All these are considered in the provided analysis and shown in the DTMC of Figure 1, which refers to each access category separately. Note that the state $\{i, 1,0,0\}$ is missing.

The other two symbols are $j$ for the backoff stage described above and $k$ which accounts for the backoff delay and takes values $k \in\left[0,1, \ldots, W_{i, j}-2\right]$ for $w=0, k \in$ $\left[0,1, \ldots, W_{i, j}-1\right]$ for $j>0$ and $w=1$, and $k \in\left[1, \ldots, W_{i, j}-\right.$ 1] for $j=0$ and $w=1$. In [5], a similar DTMC is used for the legacy dot11. This model is extended considerably so as to include all the new characteristics of dot11e and a finite retry limit. Our analysis also deviates from [5] since the first state of the chain does not exist.

The probability $p_{i, 0}$ (or $p_{i, 1}$ ) is that another terminal's access category is transmitting after an idle period (or after a busy period), without errors. The opposite case, that the channel remains idle after an idle period, is represented by $q_{0}$ (or after a busy period $q_{1}$ ). After these explanations, all the transitions of the DTMC have been verified and the following equations are accrued:

$$
b_{i, 1, j, 0}=\psi_{i, j} b_{i, 0,0,0}
$$

for $j=1,2, \ldots, L_{i}$,

$$
b_{i, 1, j, k}=\frac{1+p_{i, 0}\left(W_{i, j}-1-k\right)}{1-p_{i, 1}} \psi_{i, j} b_{i, 0,0,0}
$$

for $k=1,2, \ldots, W_{i, j}-1$ and $j=0, \ldots, L_{i}$,

$$
b_{i, 0, j, k}=\left(W_{i, j}-1-k\right) \psi_{i, j} b_{i, 0,0,0}
$$


for $k=0,1, \ldots, W_{i, j}-2$ and $j=1, \ldots, L_{i}$, where

$$
\psi_{i, j}= \begin{cases}\frac{1}{W_{i 0}-1}, & j=0, \\ \frac{p_{i, 0}}{W_{i, 1}}, & j=1, \\ \frac{p_{i, 0}}{W_{i, 1}} \Pi_{i, j}, & j=2,3, \ldots, m_{i}, \\ \frac{p_{i, 0}}{W_{i, 1}} \Pi_{i, m_{i}} P_{i, j}, & j=m_{i}+1, \ldots, L_{i} .\end{cases}
$$

$\Pi_{i, j}$ and $P_{i, j}$ are defined as

$$
\begin{aligned}
\Pi_{i, j} & =\prod_{x=2}^{j}\left[\frac{p_{i, 1}}{W_{i, x}}+\frac{p_{i, 0}}{W_{i, x}}\left(W_{i, x-1}-1\right)\right], \\
P_{i, j} & =\prod_{x=m_{i}+1}^{j}\left[\frac{p_{i, 1}}{W_{i, m_{i}}}+\frac{p_{i, 0}}{W_{i, m_{i}}}\left(W_{i, m_{i}}-1\right)\right] .
\end{aligned}
$$

Applying the normalization condition for each access category's DTMC, as each exponential backoff algorithm runs independently, we have

$$
\begin{aligned}
& \sum_{k=0}^{W_{i, 0}-2} b_{i, 0,0, k}+\sum_{k=1}^{W_{i, 0}-1} b_{i, 1,0, k} \\
& \quad+\sum_{j=1}^{L_{i}}\left[\sum_{k=0}^{W_{i, j}-2} b_{i, 0, j, k}+\sum_{k=0}^{W_{i, j}-1} b_{i, 1, j, k}\right]=1 .
\end{aligned}
$$

After solving this equation, $b_{i, 0,0,0}$ is found as

$$
\begin{aligned}
b_{i, 0,0,0}= & \frac{2\left(1-p_{i, 1}\right)}{K_{i}+\Lambda_{i}}, \\
K_{i}= & W_{i, 0}\left(1-p_{i, 1}\right)+p_{i, 0}\left(W_{i, 1}-1\right)\left(2-p_{i, 1}\right) \\
& +2 p_{i, 0}\left(W_{i, 0}-2\right)+4, \\
\Lambda_{i}= & \sum_{j=2}^{L_{i}} \psi_{i, j} W_{i, j}\left[\left(W_{i, j}-1\right)\left(1-p_{i, 1}+p_{i, 0}\right)+2\right] .
\end{aligned}
$$

The probabilities of accessing the channel in a time slot, whether the previous slot was idle or busy, are given by the following equations:

$$
\tau_{i, w}= \begin{cases}\frac{\sum_{j=0}^{m_{i}} b_{i, 0, j, 0}+\sum_{j=m_{i}}^{L_{i}} b_{i, 0, j, 0}}{P_{\text {idle, }}}, & w=\text { idle }, \\ \frac{\sum_{j=1}^{m_{i}} b_{i, 1, j, 0}+\sum_{j=m_{i}}^{L_{i}} b_{i, 1, j, 0}}{1-P_{\text {idle }}}, & w=\text { busy }\end{cases}
$$

where $P_{\text {idle }}$ is derived by the solution of $P_{\text {idle }}=q_{0} P_{\text {idle }}+q_{1}(1-$ $\left.P_{\text {idle }}\right)$, and describes the probability that the channel is idle in the previous time slot (take notice that this is different from the current idle slot symbolized below as $\left.P_{\text {idle }}\right)$.

\subsubsection{Successful transmission probability}

The probabilities that the channel remains idle after an idle (or busy) time slot can be found in a straightforward manner by supposing that no other station transmits in that time slot:

$$
q_{w}=\prod_{i=0}^{3}\left(1-\tau_{i, w}\right)^{N_{i}}
$$

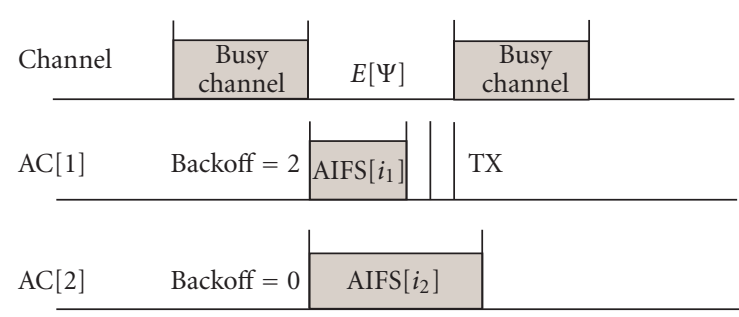

FIGURE 2: The AIFS differentiation prevents a collision that otherwise would have happened.

The probability of another AC transmitting is relatively complex. Except from the other station's AC transmition, an intercollision handler and virtual collision handler must also be taken into account. In the proposed analysis, such a collision handler is also implemented, adding as well as a correlation measure which gives a close approximation of the intercollision problem.

The phenomenon of intercollision happens when two ACs have different AIFS, and the one with the higher AIFS and higher $E[\Psi]$ has a smaller backoff value. Thus it may happen that these ACs will collide and the differentiation offered from the use of AIFS will be lost. see Figure 2:

$$
r\left(i_{1}, i_{2}\right)=\max \left[1-\frac{\operatorname{AIFS}\left[i_{1}\right]-\operatorname{AIFS}\left[i_{2}\right]}{E[\Psi]}, 0\right], \quad i_{1} \geq i_{2},
$$

where $E[\Psi]$ is the mean consecutive number of idle slots. The notation min is used to maintain the accuracy of the model:

$$
E[\Psi]=\min \left(\frac{P_{\text {idle }}}{1-P_{\text {idle }}}, 1\right) .
$$

This specific correlation measure simplifies the analysis, because it does not increase the complexity of the mathematical analysis when trying to solve the DTMC. Therefore, the probabilities of a transmission failure (taking into account the collision probability and error probabilities) after an idle or busy slot are

$$
\begin{aligned}
p_{i, 0}= & 1-\prod_{z<i}\left(1-\tau_{z, \text { idle }}\right)^{\left\lfloor N_{z} \cdot r(z, i)\right\rceil} \\
& \times\left(1-\tau_{i, \text { idle }}\right)^{N_{i}-1} \prod_{z>i}^{3}\left(1-\tau_{z, \text { idle }}\right)^{N_{z}}, \\
p_{i, 1}= & 1-\left(1-\tau_{i, \text { busy }}\right)^{N_{i}-1} \prod_{z>i}^{3}\left(1-\tau_{z, \text { busy }}\right)^{N_{z}} .
\end{aligned}
$$

The successful transmission probability in a time slot of an $\mathrm{AC}$ is

$$
\begin{aligned}
P_{s, i}= & P_{\text {idle }} \cdot N_{i} \cdot \tau_{i, \text { idle }} \cdot \prod_{z<i}\left(1-\tau_{z, \text { idle }}\right)^{\left\lfloor N_{z} \cdot r(z, i)\right\rceil} \\
& \times\left(1-\tau_{i, \text { idle }}\right)^{N_{i}-1} \cdot \prod_{z>i}\left(1-\tau_{z, \text { idle }}\right)^{N_{z}} \\
& +\left(1-P_{\text {idle }}\right) \cdot N_{i} \cdot \tau_{i, \text { busy }} \cdot\left(1-\tau_{i, \text { busy }}\right)^{N_{i}-1} \\
& \times \prod_{z>i}\left(1-\tau_{z, \text { busy }}\right)^{N_{z}} .
\end{aligned}
$$




\subsubsection{Mean backoff duration}

$E[\mathrm{BD}]_{i}$ is defined as the mean backoff delay, which is the sum of the backoff transitions $E[X]_{i}$ when the channel is idle, and the delay due to freezing $E[F]_{i}$, all of which referring to each $\mathrm{AC}$ :

$$
E[\mathrm{BD}]_{i}=E[X]_{i} \sigma+E[F]_{i} .
$$

The backoff transition delay is $E[X]_{i}$ defined as the number of slot times $k$ that are needed for the AC to reach state 0 and transmit, considering that the counter is at the state $b_{i, 1, j, k}$ or $b_{i, 0, j, k}$. The number of times the counter is stopped (freezes) is not taken into account, as they are calculated separately in (20)

$$
E\left[X_{i}\right]=\frac{\sum_{j=0}^{L_{i}} \sum_{k=0}^{W_{i, j}-2} k b_{i, 0, j, k}}{P_{\text {idle }}} .
$$

After some algebra, the backoff transition delay is found as

$$
\begin{aligned}
E[X]_{i} & =\frac{b_{i, 0,0,0} \cdot M_{i}}{12 P_{\text {idle }}}, \\
M_{i} & =\sum_{j=1}^{L_{i}} \psi_{i, j}\left(W_{i, j}-1\right)\left(W_{i, j}-2\right)\left(4 W_{i, j}-3\right),
\end{aligned}
$$

and the delay due to freezing of the backoff counter is calculated as follows. Note that the denominator of $E[F]_{i}$ is the exact opposite of the denominator of $E[X]_{i}$ :

$$
E[F]_{i}=\frac{E\left[N_{f}\right]_{i}}{1-P_{\text {idle }}}\left[\sum_{i=0}^{3} P_{s, i} T_{s, i}+P c T_{c, i}\right],
$$

where $E\left[N_{f}\right]_{i}$ is the number of freezes, and it is analyzed as the fraction of the mean value of the counter $E[X]_{i}$ divided with the mean consecutive number of idle slots, defined in (13).

In order to find the MAC delay, the mean delay must be subtracted from the dropping delay defined as

$$
\begin{aligned}
E[\text { Drop }]_{i}= & b_{i, 0,0,0}\left(T_{c}+T_{\text {protect }}\right) \psi_{i, L_{i}} \\
& \times\left[\frac{1+p_{i, 0}\left(W_{i, L_{i}}-1\right)}{1-p_{i, 1}} p_{i, 1}+\left(W_{i, j}-1\right) p_{i, 0}\right] .
\end{aligned}
$$

\subsection{Elementary conditional probability analysis (ECPA)}

The proposed probabilistic analysis is simpler than the previous solution of DTMC, because it is based on conditional probabilities of each access category independently [10]. Two events are defined here. The first is called $T X_{i}$ and means that a station's AC is transmitting a frame into a time slot, and the second is $s=j$ is that the station's AC is in backoff stage $j$, where $j \in\left[0, L_{i}\right]$, whenever $L_{i}$ is different in basic and RTS/CTS method according to the short and long retry limit. From Bayes' theorem, we have

$$
P\left(T X_{i}\right) \frac{P\left(s=j \mid T X_{i}\right)}{P\left(T X_{i} \mid s=j\right)}=P\left(s_{i}=j\right) .
$$

\subsubsection{Successful transmission probability}

From [10, equations (2)-(7)], with amendments so as to include the four ACs $(i=\{0,1,2,3\})$, we have that the transmission probability can be written as

$$
\tau_{i}=\frac{1}{\left(\left(1-p_{i}\right) /\left(1-p_{i}^{L_{i}+1}\right)\right) \sum_{j=0}^{L_{i}} p_{i}^{j} \cdot\left(1+E[\mathrm{BD}]_{i, j}\right)} .
$$

In order to include the freezing of the backoff counters, a distinction must be made. The interruption of the backoff period of the tagged station can occur by three different events and is analyzed as follows. The first is the collision of two or more stations, the second is the transmission of a single station other than the tagged one, and the third is the transmission of a single station. $p_{i}$ is the probability that the tagged station is interrupted the transmission of any other station (one or more) being

$$
p_{i}=1-\prod_{z \geq i}\left(1-\tau_{z}\right)^{N_{m, z}}
$$

and $N_{m, z}=N_{z}-\delta_{m, z}\left(\delta_{m, z}\right.$ is the Kronecker function [15]).

The probability that the tag station is interrupted by the transmission of a single station (one exactly) is given by

$$
p_{i}^{\prime}=\left(\begin{array}{c}
N_{i}-1 \\
1
\end{array}\right) \cdot \tau_{i} \cdot\left(1-\tau_{i}\right)^{N_{i}-2} \prod_{z>i}\left(1-\tau_{z}\right)^{N_{z}}
$$

\subsubsection{Mean backoff duration}

In order to find the mean backoff duration, the duration of each exponential backoff must be found, which should include the finite limit of $C W-H[j-1]$ and the freezing of backoff counter each time the slot is detected busy. For example, if there were $k$ freezings, then the delay would be $E[\mathrm{SD}]_{i, j}=\sum_{k=0}^{C W_{i}-H[j-1]}\left(k p^{k}\right) \cdot\left(1-p_{i}\right)$, which gives finally

$$
E[\mathrm{SD}]_{i, j}=\frac{C W_{i}-H[j-1]}{2 \cdot\left(1-p_{i}\right)} .
$$

Taking into account all the possible series of the exponential backoff, the mean backoff duration is given from

$$
E[\mathrm{BD}]_{i, j}= \begin{cases}\sum_{k=0}^{C W_{,}-1} \frac{\mathrm{BD}_{i, j}^{k}}{C W_{i, j}}, & 0 \leq j \leq m_{i}, \\ E[\mathrm{BD}]_{i, m_{i}}, & m_{i} \leq j \leq L_{i} .\end{cases}
$$

\subsection{Queuing network (QN) model analysis}

This analysis is based on the Choi et al. [11] queuing model. In our model, the approach towards the network is different than any one proposed before, because it models the behavior of each AC, which contains $N_{i}$ stations instead of a single station, independently (see Figure 3). Except from that, each backoff stage is modeled by a $G / G / \infty$ queuing system. 

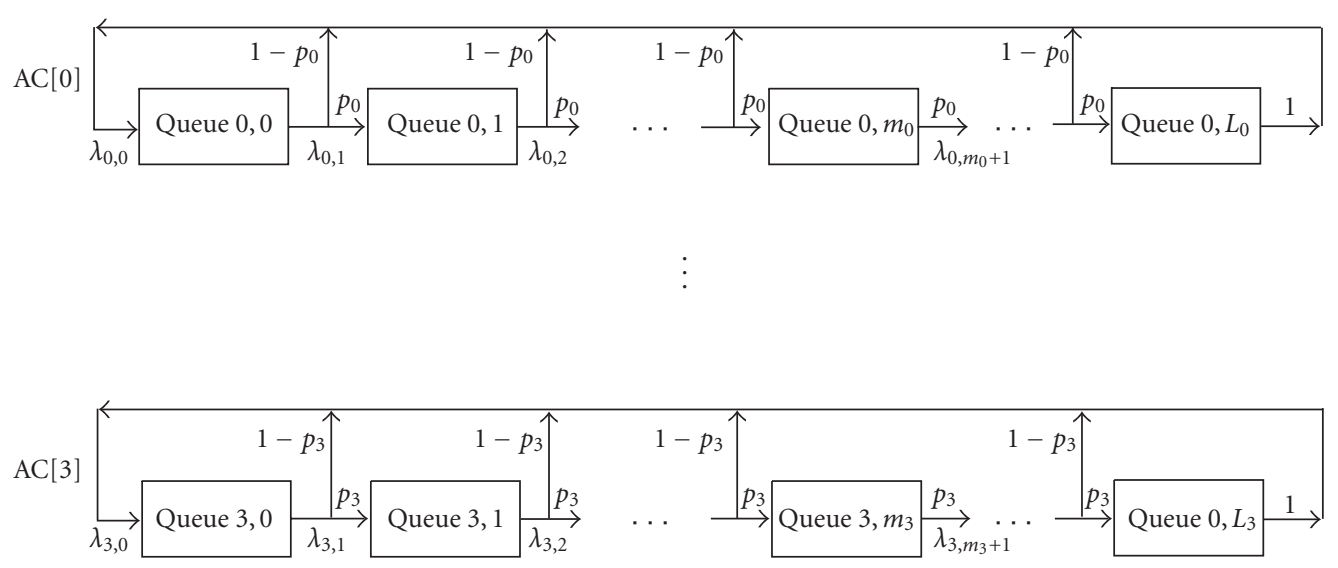

FIGURE 3: Queuing network model and analyzing the backoff duration with $Z$-transform.

An infinite number of parallel servers are used so that each queue can serve all stations simultaneously without queuing delay. In addition, the queuing delay is found by taking into account the freezing of backoff counters. Similar to the previous two models, the first queue has less length than the other ones. This solution is based on the assumption that the transmission probability can be expressed as the total attempt rate $\lambda_{i}$, divided by the number of stations of each AC independently:

$$
\tau_{i}=\frac{\lambda_{i}}{N_{i}}
$$

Let us define $\lambda_{i, j}$ as the arrival rate and $\mu_{i, j}$ as the average service rate, at each queue of each AC, where $\mu_{i, k}$ is found from the backoff duration of each queue, which is calculated from the $Z$-transform of each queue given below. From Little's theorem, the number of stations in each queue and in each $\mathrm{AC}$ can be found by

$$
N_{i, j}=\frac{\lambda_{i, j}}{\mu_{i, j}} .
$$

The transition probability from one queue to the next one is related the arrival rates. However, it should be noted that a small difference is found from queue 0 to queue 1 , as it has been explained that the value 0 of the first backoff window is not chosen:

$$
\lambda_{i, j+1}=p_{i} \lambda_{i, j}, \quad j=0, \ldots, L_{i}-1,
$$

where the total attempt rate $\lambda_{i}$ is given by

$$
\lambda_{i}=\sum_{j=0}^{L_{i}} \lambda_{i, j}=\lambda_{i, 0} \frac{1-p_{i}^{L_{i}+1}}{1-p_{i}}
$$

and the average service rate of each queue is found from

$$
\mu_{i, j}=\frac{1}{1+E[\mathrm{BD}]_{i, j}} .
$$

The reason for adding 1 with $E[B D]_{i, j}$ is that to get out of the queue one more slot is required, corresponding to transmission.

\subsubsection{Successful transmission probability}

Having calculated $\lambda_{i, j}$ and $\mu_{i, j}$, we can use again Little's theorem:

$$
N_{i}=\sum_{j=0}^{L_{i}} N_{i, j}=\lambda_{i, 0} \sum_{j=0}^{L_{i}} p_{i}^{j}\left(1+E[\mathrm{BD}]_{i, j}\right) .
$$

In (33), the sum is too complicated to be solved and it needs computer mathematical tools. Finally $\tau_{i}$ is computed from (28):

$$
\tau_{i}=\frac{\lambda_{i}}{N_{i}}=\frac{1}{\left(\left(1-p_{i}\right) /\left(1-p_{i}^{L_{i}+1}\right)\right) \sum_{j=0}^{L_{i}} p_{i}^{j} \cdot\left(1+E[B D]_{i, j}\right)} .
$$

From the above mathematical results, we can see that (23) and (34) are the same. So both types of solutions give similar results. Thus to find the probability of successful transmission in both models, we use

$$
P_{s, i}=N_{i} \cdot \tau_{i} \cdot \prod_{z}\left(1-\tau_{z}\right)^{N_{m, z}} .
$$

\subsubsection{Mean backoff duration}

The mean backoff duration is similar to the one analyzed in the ECPA analysis.

\section{THROUGHPUT AND DELAY}

\subsection{Saturation throughput}

\subsubsection{Block-ACK disabled}

The saturation throughput for every $\mathrm{AC}$ and for packets with mean length $E[L]$ is given by

$$
S_{i}=\frac{p_{e, i} P_{s, i} E[L]}{T_{\text {slot }, i}},
$$

where

$$
T_{\text {slot }, i}=P_{\mathrm{idle}} \sigma+\sum_{i=0}^{3}\left[\left(1-p_{e, i}\right) P_{s, i} T_{s, i}\right]+\left(P c+p_{e, i} P_{s, i}\right) T_{c, i} .
$$




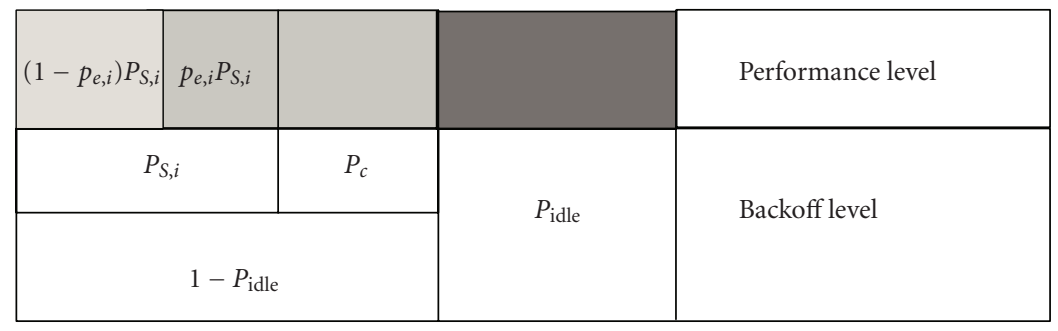

FIGURE 4: Probability spaces in the analysis of 802.11 standards for error behavior.

As shown in Figure 4, the probability of error affects the successful transmission probability only. Thus whenever both the events of successful transmission probability and error happen, they are regarded as collisions.

Since the errors are uniformly distributed, the error events are independent and identically distributed (i.i.d.) thus the frame error probability is given by

$$
p_{e, i}=\left(1-p_{e, i}^{\mathrm{data}}\right)\left(1-p_{e, i}^{\mathrm{ACK}}\right)\left(1-p_{e}^{\mathrm{RTS}}\right)\left(1-p_{e}^{\mathrm{CTS}}\right) \text {. }
$$

$p_{e, i}^{\text {data }}$ and $p_{e, i}^{\mathrm{ACK}}$ show the uniformly distributed errors in the data packet and in the acknowledgment, and the same applies for the probabilities $p_{e, i}^{\mathrm{RTS}}$ and $p_{e, i}^{\mathrm{CTS}}$ which are used only in RTS and CTS access method. If Basic access method is used, then $p_{e, i}^{\mathrm{RTS}}=p_{e, i}^{\mathrm{CTS}}=0$.

Then the collision probability is

$$
P c=1-P_{\text {idle }}-\sum_{i=0}^{3} P_{s, i}
$$

We must also mention that whenever the retry limit is reached, the packet is dropped. However such a probability is included in $P c$, and the retransmissions required after a collision or a drop are based on the upper layer and do not affect the performance of the studied MAC layer.

\subsubsection{Block-ACK enabled}

Another characteristic of the IEEE 802.11e standard is the Block-ACK feature, which is not obligatory. However BlockACK can mitigate the overhead problem, especially in higher data rates which are supported by the forthcoming $802.11 \mathrm{n}$. Data rates of nearly $432 \mathrm{Mbps}$ tend to have $10 \%$ of MAC efficiency [14].

The Block-ACK feature allows a number of data units to be transmitted and afterwards the sender sends a BlockACK request (BAR) and receives a Block-ACK (BA) frame. Throughput is increased since less ACK frames are used for a transmission. Analysis of the Block-ACK scheme (BTA) is not within the scope of the paper and more information can be found in the standard [2]. The problem with errors in the BTA scheme is similar to the RTS/CTS and requires to change all of the above equations which include errors in RTS and CTS frames with errors in BAR and BA frames, and to make all the respective errors of ACK equal to zero. However since the errors are uniformly distributed, the probability of error in one of these packets is equal. Finally

$$
S_{i}^{\prime}=\frac{\left(1-p_{e, i}\right) \cdot P_{s, i}^{\prime} \cdot F \cdot E[L]}{P_{\text {idle }} \sigma+\sum_{i=0}^{3}\left(1-p_{e, i}\right) P_{s, i}^{\prime} T_{s, i}+\left(P c+p_{e, i} P_{s, i}\right)} .
$$

The time for successful transmission $T_{s, i}$ is thus much bigger since it includes $F$ frames and SIFS time, plus the exchange of the BAR and BA. Moreover $H$ is the physical layer header and $\delta$ is the transmission delay:

$$
\begin{aligned}
T_{s, i}^{\text {basic }}= & T_{E, i}=F \cdot(H+E[L]+\operatorname{SIFS}+\delta)+\operatorname{AIFS}[i]+H \\
& +T_{\mathrm{BAR}}+\operatorname{SIFS}+\delta+H+T_{\mathrm{BA}}+\delta, \\
T_{c, i}= & F \cdot(H+E[L]+\operatorname{SIFS}+\delta)+\operatorname{EIFS}[i]+H+T_{\mathrm{BAR}}+\delta,
\end{aligned}
$$

where $\operatorname{EIFS}[i]=\operatorname{SIFS}+H+T_{\mathrm{BA}}+\operatorname{AIFS}[i]$.

\subsection{MAC delay}

\subsubsection{Mean value of the MAC delay}

In 802.11e [2], two different access mechanisms are provided. The first one is the use of acknowledgments by ACKs (called here "basic") and the other by transmitting request-to-send and clear-to-send packets. The transmission times $T_{s, i}^{\text {Basic }}$ and $T_{s, i}^{\mathrm{RTS} / \mathrm{CTS}}$, and the times $T_{c, i}^{\mathrm{Basic}}$ and $T_{c, i}^{\mathrm{RTS} / \mathrm{CTS}}$ for a collision can be found in [6].

The mean delay can be defined for each AC by the following equation:

$$
\begin{aligned}
E[D]_{i}= & E\left[N_{c s}\right]_{i}\left(E[\mathrm{BD}]_{i}+T_{c}+T_{\text {protect }}\right) \\
& +E[\mathrm{BD}]_{i}+T_{s, i} .
\end{aligned}
$$

The first part of the equation is the delay due to consecutive unsuccessful transmissions, the second part is the mean backoff delay, whenever this transmission will be completed, and the third part is the transmission duration. All are referred to each AC. Following (42), $E\left[N_{c s}\right]_{i}$ can be defined as the mean number of collisions that are followed by a successful transmission:

$$
E\left[N_{c s}\right]_{i}=\frac{1-P_{\mathrm{idle}}-P_{s, i}}{P_{s, i}} .
$$




\subsubsection{PMF of the MAC delay}

Having supposed that the standard refers to an integer number of time slots, then the $Z$-transform can be used to calculate the delay. $Z$-transform is well used in the analysis of queuing systems, because its derivatives can provide measures such as mean values, variances, and some other possible moments of the PMF of the MAC delay.

In order to include the freezing of the backoff counters, a distinction has been made in (24) and (25).

The probability that the tag station is interrupted by the transmission of a single station (one exactly) is given by

$$
p_{i}^{\prime}=\left(\begin{array}{c}
N_{i}-1 \\
1
\end{array}\right) \cdot \tau_{i} \cdot\left(1-\tau_{i}\right)^{N_{i}-2} \prod_{z>i}\left(1-\tau_{z}\right)^{N_{z}} .
$$

The phenomenon that the slot is interrupted from a collision or a successful transmission is described by

$$
\begin{gathered}
P(\text { collision } \mid \text { slot is interrupted })=p_{c, i}=\frac{p_{i}-p_{i}^{\prime}}{p_{i}}, \\
P(\text { successful by one AC } \mid \text { slot is interrupted })=p_{t, i}=\frac{p_{i}^{\prime}}{p_{i}} .
\end{gathered}
$$

In our case, each state of backoff duration is said to have a delay $\mathrm{SD}_{i}(z)$. In order to count down to the next state, the slot must remain idle, which is symbolized by the duration of the empty in $Z$-transform multiplied by the probability of the slot to be idle, $P_{\text {idle }} Z^{\sigma}$. Hence the $Z$-transform of that delay is

$$
\mathrm{SD}_{i}(z)=\frac{P_{\mathrm{idle}} Z^{\sigma}}{1-p_{i} \cdot\left(p_{t, i} Z^{T_{s, i}}+p_{c, i} Z^{T_{c, i}}\right)} .
$$

Then the total delay of backoff duration is given from the geometric sum, since its state is chosen uniformly. Note that the first queue of each $\mathrm{AC}$ is smaller since the first state is not chosen:

$$
\mathrm{BD}_{i, j}(z)= \begin{cases}\sum_{k=0}^{C W_{i, j}-1} \frac{\mathrm{SD}_{i}^{k}(z)}{C W_{i, j}}, & 0 \leq j \leq m_{i}, \\ \mathrm{BD}_{i, m_{i}}(z), & m_{i} \leq j \leq L_{i} .\end{cases}
$$

In the previous subsection, we have shown a unified method to find the mean MAC delay for all the models. In the following subsection, the above metrics for MAC delay will correspond only to the model 3 . This happens because the solution of the DTMC after theoretically infinite retries gives mean values. Thus the $Z$-transform of the MAC delay will be given as a function of $D_{i}(z)$ :

$$
\begin{aligned}
D_{i}(z)= & \left(1-p_{i}\right) z^{T_{s, i}} \sum_{j=0}^{L_{i}}\left[\left(p_{i} z^{T_{c}}\right)^{j} \prod_{f=0}^{j} \mathrm{BD}_{i, f}(z)\right] \\
& +\left(p_{i} z^{T_{c}}\right)^{L_{i}+1} \prod_{f=0}^{L_{i}} \mathrm{BD}_{i, f}(z) .
\end{aligned}
$$

The first part signifies the correct transmission ((1 $\left.p_{i}\right) z^{T_{s, i}}$ ) having encountered a number of collisions in the previous stages, whereas the second part is the delay associated with dropping of a packet after $L_{i}+1$ retries. However, to find the mean value and the standard deviation (SD), the 1st and the 2 nd moments of the above equations must be found, respectively, and we need

$$
\begin{aligned}
E[D]_{i} & =\left.\frac{\partial D_{i}(z)}{\partial z}\right|_{z=1}, \\
\operatorname{Var}^{2}[D]_{i} & =\left.\frac{\partial^{2} D_{i}(z)}{\partial z^{2}}\right|_{z=1}+\left.\frac{\partial D_{i}(z)}{\partial z}\right|_{z=1}-\left\{\left.\frac{\partial D_{i}(z)}{\partial z}\right|_{z=1}\right\}^{2} .
\end{aligned}
$$

The last part is to find MAC delay distribution. It is well know that every $Z$-transform of the PGF can be written as

$$
D_{i}(z)=\sum_{k=0}^{\infty} d_{i, k} z^{k}
$$

It seems that from the definition $d_{i, k}$ is the inverse $Z$ transform of the $D_{i}(z)$. A method that gives the inverse $Z$-transform with a predefined error bound is the LatticePoisson algorithm [16], which is valid for $\left|d_{i, k}\right| \leq 1$. Thus the PMF (probability mass function) of the MAC delay is given by

$$
d g_{i, k}=\frac{1}{2 k r^{k}} \sum_{h=1}^{2 k}(-1)^{h} \operatorname{Re}\left(D G_{i}\left(r e^{i \pi j / k}\right)\right),
$$

where $D G_{i}(z)$ is the generating function

$$
D G_{i}(z)=\sum_{k=0}^{\infty} d g_{i, k} z^{k}=\frac{1-D_{i}(z)}{1-z} .
$$

The following values can be used: $r=-\gamma /(2 * k)$ and $\gamma=1$.

\section{COMPARISON ANALYSIS AND EVALUATION}

For validating the correctness of the mathematical analyses, OPNET modeler (version 12) was used with the EDCA simulation model incorporated. For further validation, we also compare the Xiao model [6] which is slightly alternated for reasons of fair comparison. In Table 2, the simulation parameters are summarized. The accuracy values of the figures are explained in Section 4.2.

In Figure 5, saturation throughput comparison is presented for the three models, the Xiao model, and the simulations. The throughput of ACO is twice the throughput of $\mathrm{AC} 1$, which is derived from the half $C W \min$ and $C W \max$ values (see Table 1). It seems that most models have results close to the simulation values, with the DTMC model being the most accurate one. In fact this happens due to the ability of the DTMC to capture possible parameters of the EDCA scheme of $802.11 \mathrm{~b} / \mathrm{e}$, in each time slot, rather than average values of the other models.

Similar results can be found for the RTS/CTS access method in Figure 6. However one major difference between these methods, which cannot be derived from basic rate analyses (1 Mbps), is that, as the number of nodes (load) increases, RTS/CTS can solve, except from the hidden node terminal, part of the high collision rate. Thus a hybrid system 
TABLE 2: Simulations parameters.

\begin{tabular}{ll}
\hline Simulator & OPNET modeler 12 \\
\hline Model & EDCA model august $(2006)$ \\
Standards & $802.11 \mathrm{~b} / \mathrm{e}$ and $802.11 \mathrm{a} / \mathrm{e}$ \\
Packet size & Constant 1023 bytes $($ no segm.) \\
Interarrival time & Set according to saturation conditions \\
RTS threshold & 512 bytes \\
Simulation & $100 \mathrm{~s}$ \\
Initial seed & 128 \\
Space & Square $100 \times 100($ single hop $)$ \\
Mode & No AP functionality $($ ad hoc $)$ \\
\hline
\end{tabular}

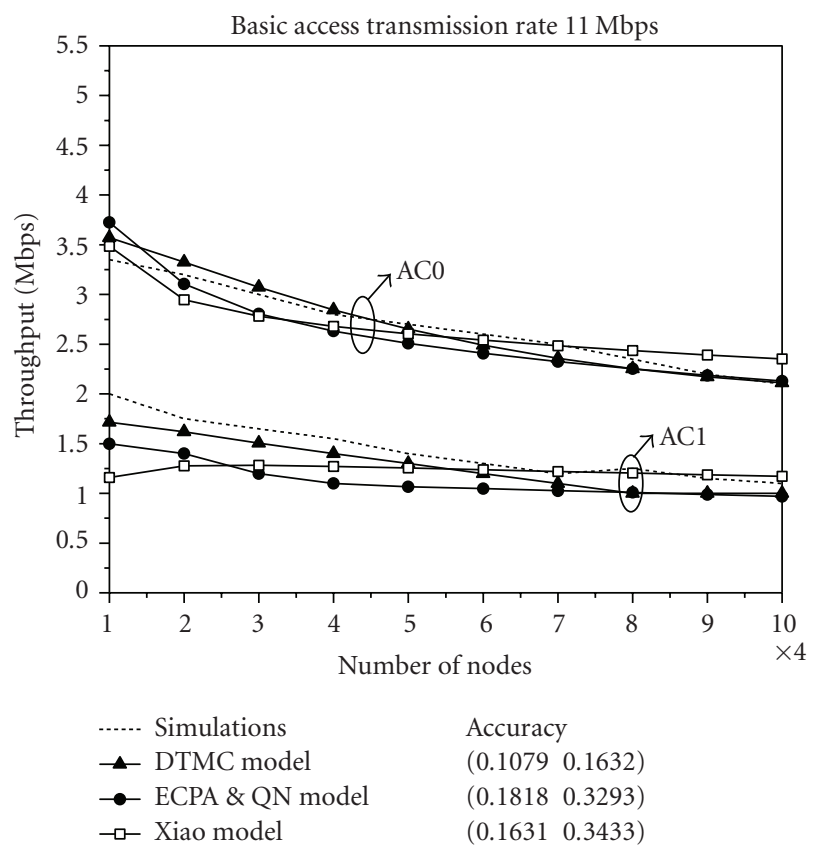

Figure 5: Model comparison in terms of saturation throughput using basic access method at $11 \mathrm{Mbps}$ transmission rate.

that would change the transmission process, according to the load, would provide higher performance of the standard.

In Figure 7, throughput is shown as a function of the number of nodes and probability of error, thus providing a $3 \mathrm{D}$ graph. The probability of error is a derivation of cross layer architectures and probabilistic nature of the channel. It is worth seeing that in RTS/CTS method, the degradation of throughput stays in very low values, and shows that RTS/CTS transmission can be a solution in erroneous environments as well. The comparison of the three models in these graphs is avoided since the degradation of the performance due to errors seems to be linear to probability of errors.

According to Figures 8 and 9, the performance analysis shows that our DTMC remains equally accurate as in throughput metric. Xiao's model tends to diverge significantly from the simulation results whereas the elementary probabilistic and queuing models seem to have a good accuracy. Moreover from the ECPA and queuing model, the first

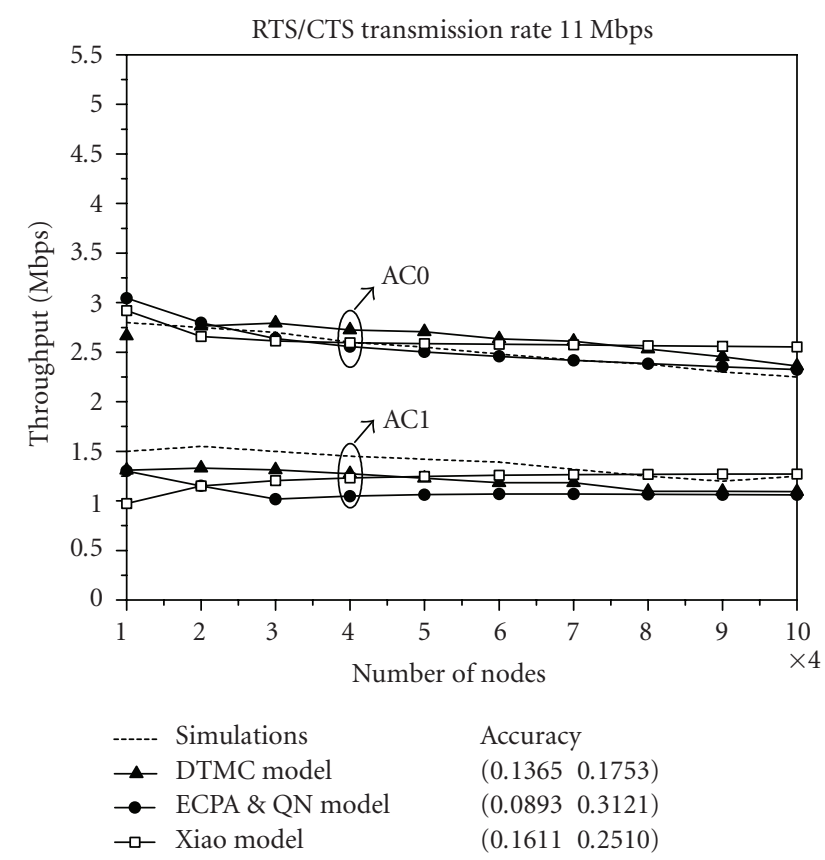

FIgURE 6: Model comparison in terms of saturation throughput using RTS/CTS access method at $11 \mathrm{Mbps}$ transmission rate.

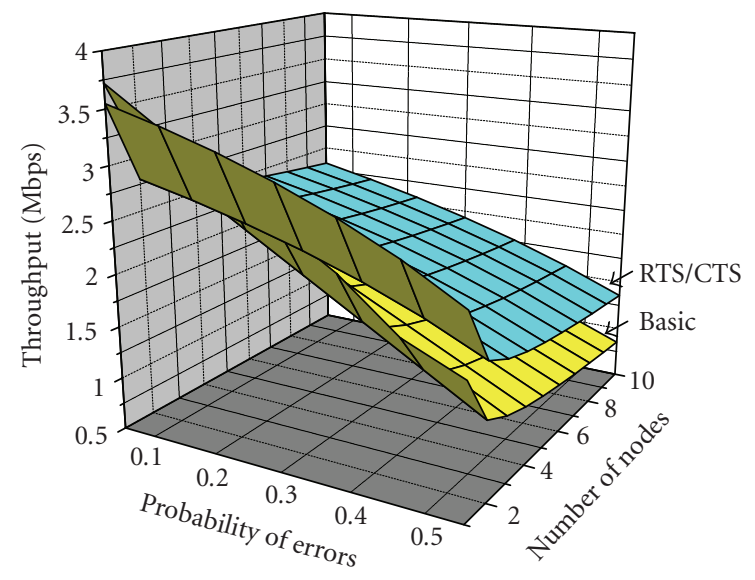

FIGURE 7: Saturation throughput at $11 \mathrm{Mbps}$, as a function of probability of error and number of stations for AC0.

derivative (signified as FD in the figures) of the $Z$-transform PGF of the MAC delay, shows the mean value. Such a mean value seems to be more accurate than the mean values provided by the other models, because it differentiates the freezing probability in collision or busy channel due to a transmission. Similarly, taking the second derivative of the PGF of the $Z$-transform, the variance is found as shown in Figure 10. Variance of the MAC delay is a significant metric, since it signifies the jitter of the multimedia traffic transmitted over the standard using $11 \mathrm{Mbps}$ bandwidth.

In Figure 12, IEEE 802.11a/e is modeled with a bandwidth of $24 \mathrm{Mbps}$ (analytical and simulation set) and as it is seen when enabled, the Block-ACK mechanism can offer higher throughput in higher load and can even provide 


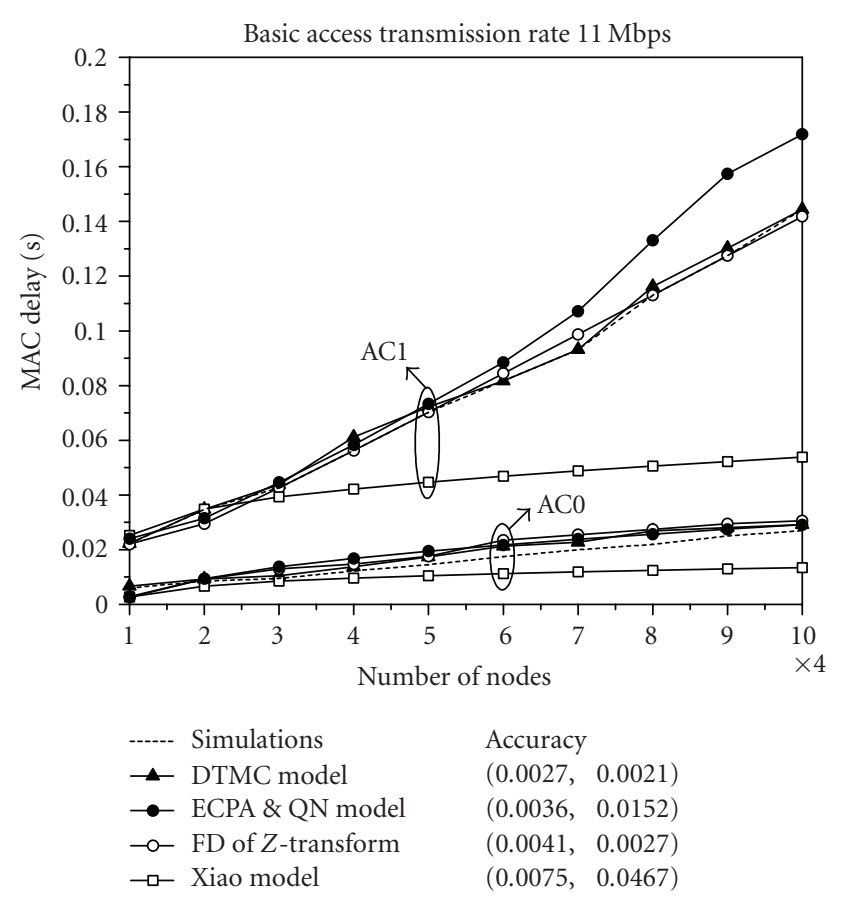

FIgURe 8: Analysis of MAC delay of the two higher ACs under saturation condition and basic access mode.

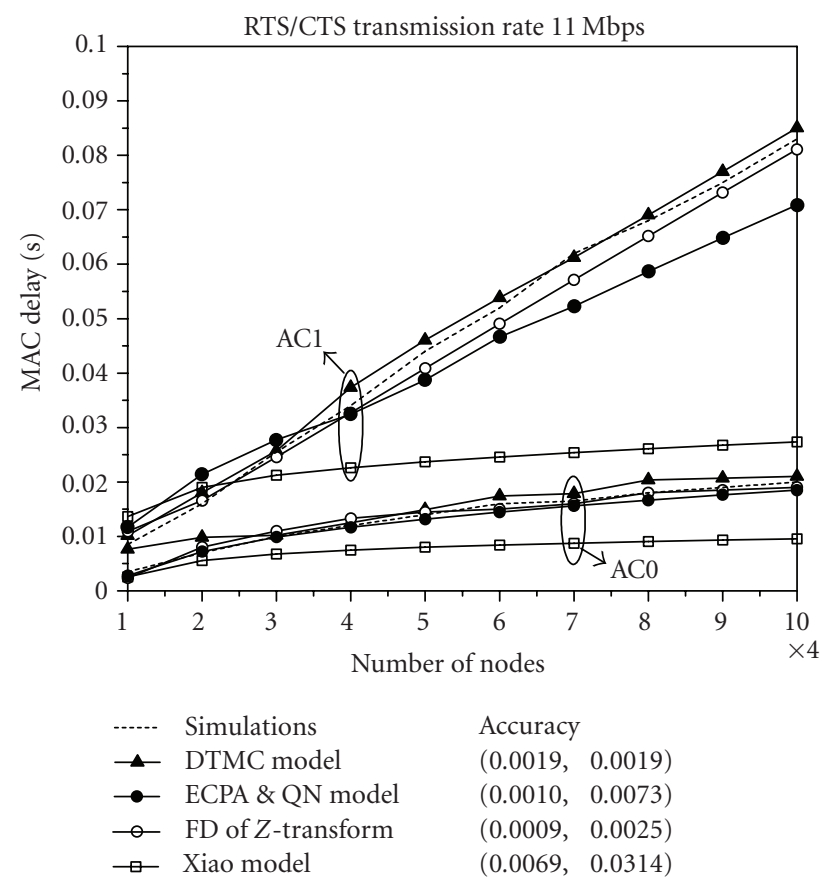

FIgURE 9: Analysis of MAC delay of the two higher ACs under saturation condition and RTS/CTS access mode.

better results in higher bandwidth occasions. This is due to the reduction of unnecessary ACKs. The reason for modeling IEEE 802.11a is that higher bandwidths are going to be used in 802.11n with Physical (PHY) and MAC layers that do not change significantly. (In Figure 12, accuracy measure-
TABLE 3: Big-O notation for computational complexity of the compared models.

\begin{tabular}{lccc}
\hline & $\tau$ & $E[\mathrm{BD}]$ & Both $\tau$ and $E[\mathrm{BD}]$ \\
\hline DTMC & Com1 & Com2 & Com2 \\
ECPA & Com3 & Com3 & Com3 \\
Queuing & Com3 & Com3 & Com3 \\
\hline
\end{tabular}

ments are not taken because the graph is not provided for depiction of Block-ACK-Section 3.1.2.)

\subsection{Complexity analysis}

Complexity is an important characteristic as regards mathematical analysis and algorithms. Comparing the three approaches in terms of complexity allows for an insight in to the usability and scalability of each one.

The DTMC model is obviously the most complex one. This is due to the independence of each state, which models a state of the BEB, and the correlation with the state of the previous slot. However the state of the previous slot is hardly incorporated in models based on queuing theory or geometric distribution since it does not allow the flexibility to change backoff duration according to the simulation needs. A significant drawback of the proposed DTMC is that nonsaturation throughput analysis becomes a complex problem, whereas in the other analyses, the arrival rate could be changed very easily with simple algebra. On the other hand, the modeling of independent states makes easier to provide amendments in the analysis, such as the one given with the inexistence of the first state. Thus it is easy to observe that the analysis of this first model requires big DTMC and more mathematical formulas to be calculated.

Moreover, the addition of extra features and the incorporation of realistic modeling in this approach inject even more complexity in the final calculations. Apart from this heuristic approach, a computational complexity comparison can be performed in terms of big- $O$ notation. Instead of computer instructions, we use a simple formula calculation as the basic unit of complexity. Each algorithm's order of complexity can be estimated as a function of the number of calculation points $N$, the number of steps used in the fixed point iteration method $M$, the retry limits $L_{i}$, and the number of ACs calculated $i$. In Table 3 , the results show that all three algorithms have linear complexity relative to $M$ and $N$, and that the DTMC model is approximately four times more complex than the other two approaches:

$$
\begin{aligned}
& \text { Com1 }=O\left[N M\left(\sum_{i}\left(4 L_{i}+1\right)\right)\right], \\
& \text { Com2 }=O\left[N\left(M\left(\sum_{i}\left(4 L_{i}+1\right)\right)+\sum_{i} L_{i}\right)\right], \\
& \text { Com3 }=O\left[N M\left(\sum_{i}\left(L_{i}+3\right)\right)\right] .
\end{aligned}
$$

Ordinary values for the parameters are: $N=10, M=20$, $L=7$, and $i=4$. 
TABLE 4: Metamodel comparison.

\begin{tabular}{lccc}
\hline & DTMC & ECPA & Queue \\
\hline Accuracy & High & Medium & Medium \\
Flexibility & High & Medium & Medium \\
Complexity & High & Low & Low \\
Nonsat & Low & Medium & Low \\
Depiction & High & Low & Medium \\
\hline
\end{tabular}

\subsection{Accuracy analysis}

In order to prove the accuracy of each model, we have used the $L^{2}$ distance, which is defined as the distance

$$
\begin{aligned}
& d\left(m_{\mathrm{sim}, N_{i}}(\min q), m_{\mathrm{anal}, N_{i}}\right) \\
& =\sqrt{\frac{1}{F N} \sum_{N_{i}=0}^{F N}\left(m_{\mathrm{sim}, N_{i}}(\min q)-m_{\mathrm{anal}, N_{i}}\right)^{2},}
\end{aligned}
$$

where $m_{\mathrm{anal}, \mathrm{N} i}$ is the analytical metric (throughput or delay) of each model, $m_{\mathrm{sim}, \mathrm{Ni}}(\min q \%)$ is the simulation of each metric (we mention it as a function of ( $\min q \%$ ) which is the closest \% quartile-in our case mean values of simulation have been used), both as functions of $N_{i}$, which is the number of nodes of each AC.

$F N$ is said to be the maximum number of nodes of each AC, defined for case $F N=10$. Thus for the comparison analysis, we have the distances, as written in Figures 5, 6, 9, and 10 . It is readily seen that the DTMC model is more accurate than the other two models, since it can capture the freezing of the backoff counter, and the effect of the previous slot to the current one. In addition, the first derivative of the MAC delay diverges the freezing probability to busy period due to a transmission or collision, and gives the best possible accuracy.

\section{DISCUSSION AND CONCLUSION}

The purpose of this work is threefold. The first goal is to present a comparison analysis of the most known analyses in order to find the best method to numerically analyze the standard, while setting the pace for future methods of analysis. Furthermore the modeling techniques, based on complexity and accuracy theory, have not been studied before in wireless networks and they could be a field of great interest, since the computer resources are finite.

The second goal was to extend the already known analyses, introducing features that change considerably the performance analysis. The combination of such features optimizes the MAC protocol outcomes, and makes each mathematical analysis avant-garde by itself.

The third goal was to correct the IEEE 802.11e from general misunderstandings, such as the phenomenon of not providing instant access after a successful transmission and the freezing of backoff counter.

From the evaluation part of the proposed work, the performance (of general BEB algorithms-numerous standards implement BEB) and simulation conclusions are as follows.

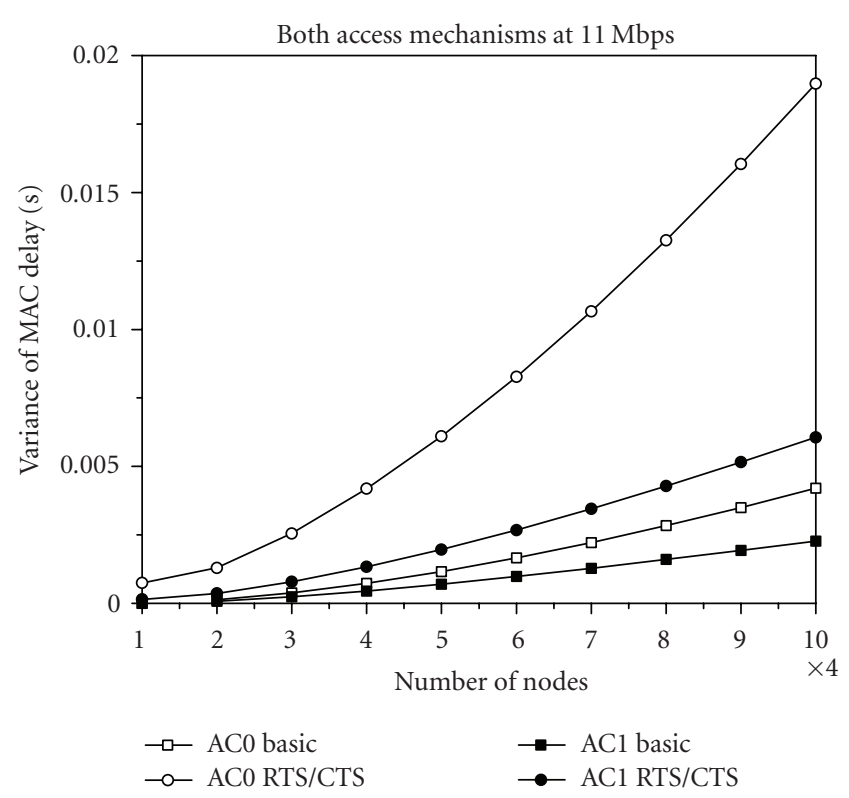

FIGURE 10: Jitter (second derivative of $Z$-trasform) is shown for the $\mathrm{ACO}$ and $\mathrm{AC1}$, and for variable number of stations in each AC. The transmission rate is at $11 \mathrm{Mbps} 802.11 \mathrm{~b} / \mathrm{e}$.

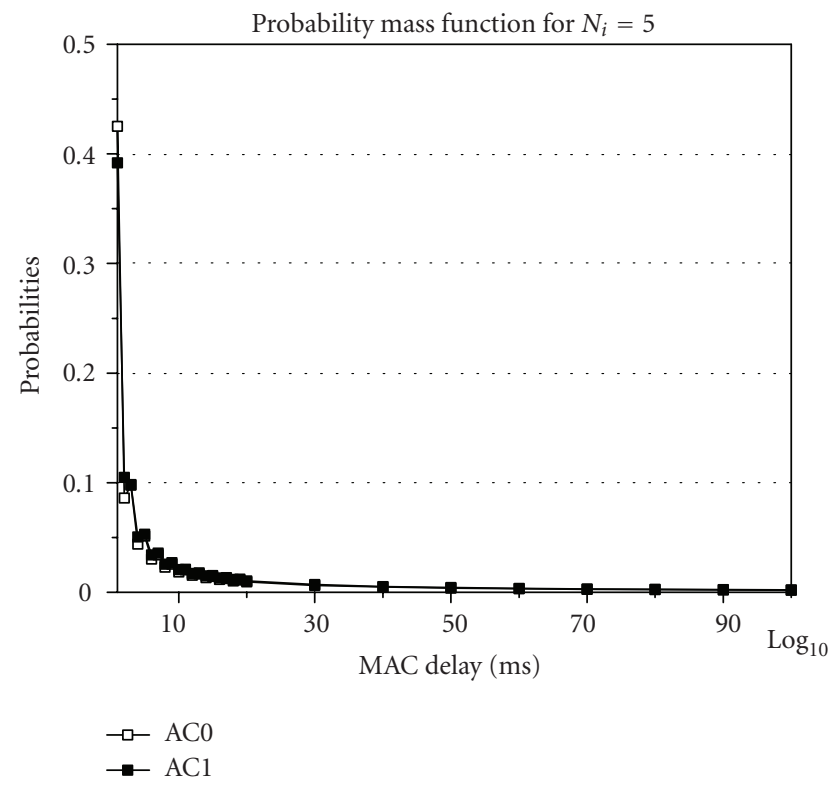

FIgURe 11: Probability mass function (PMF) for basic access mode and for both ACs. The transmission rate is at $11 \mathrm{Mbps} 802.11 \mathrm{~b} / \mathrm{e}$ and the graphs are for 5 numbers of states at each $\mathrm{AC}[i]$.

\section{(1) Performance conclusions}

(i) In order to have better performance in exponential backoff algorithms, methods that provide fairness of transmission (such as RTS/CTS), apart from aiding in the hidden terminal problem, can reduce the collision rate and improve the performance in erroneous environment. Thus it could be used in WiFi implementations over rural areas. 


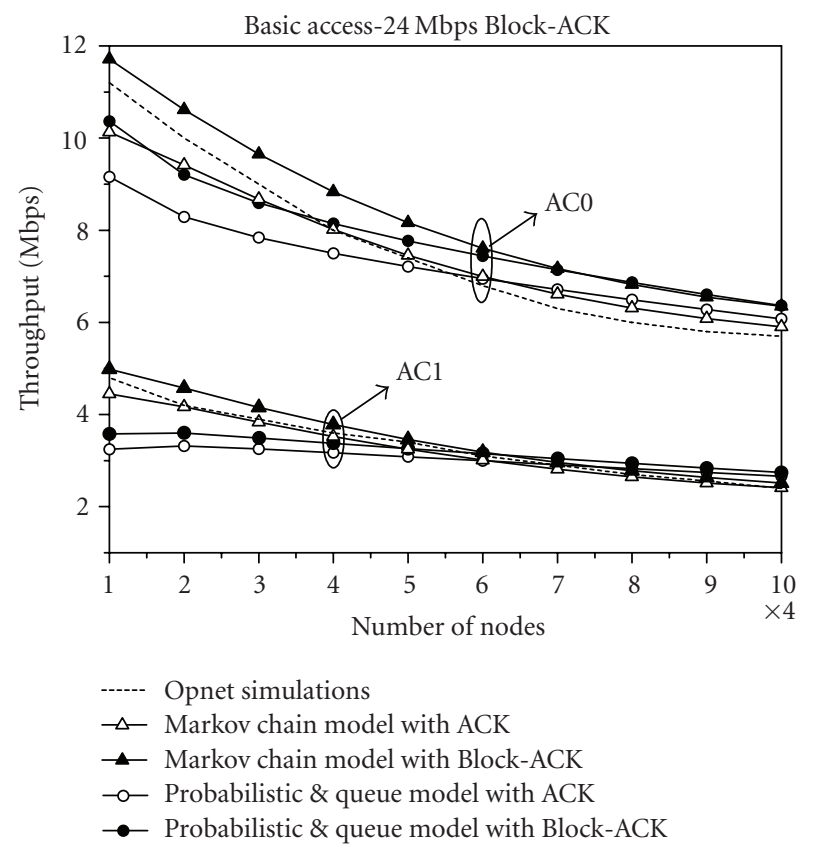

FIgURE 12: Model comparison of saturation throughput with Block-ACK being enabled $(\mathrm{F}=64)$ in $24 \mathrm{Mbps}$ with IEEE 802.11a.

(ii) RTS/CTS and similar access methods should be used only in higher loads, because in lower ones they tend to diminish the throughput performance.

(iii) Analogies on initial windows (e.g., CWmin) in BEB algorithms can give the similar analogies on throughput performance.

(iv) Methods that group packets (e.g., Block-ACK or TxOP) can augment the throughput performance.

\section{(2) Simulation conclusions}

As regards the accuracy, the DTMC model offers better results, owing to the fact that more EDCA characteristics can be included. This leads to another advantage of the DTMC model which is its flexibility. The modeling of each independent state allows for extreme detail in modeling each specific characteristic of the MAC protocol, such as the absence of the first state and the correlation of each state with the previous slot. Moreover the DTMC can also be used as a depiction of the states of the MAC protocol.

On the other hand, the other two models demonstrate different advantages. They lead to approximate results bearing less complexity compared to the DTMC model, both having the same accuracy and small differences in complexity. Moreover they allow for nonsaturation conditions of traffic, whereas in the DTMC model case, this can prove to be a very complex issue.

The $Z$-transform is used as a method to calculate the accurate delay distribution, while having different types of queues, depicting the heterogeneity of the multimedia applications. Even if such a combination of features and corrections increase, the complexity of the proposed analyses, it is the price that must be paid for the improved accuracy and re- alism, especially when new machines can solve problems in acceptable time lengths. OPNET modeler 12, was used in two ways: first to verify the correctness of analyses and second as a tool to calculate the accuracy analysis. In Table 4, the above are summarized. Fading channels can be used in order to optimize MAC layer metrics through cross-layer techniques in high-mobility scenarios.

\section{REFERENCES}

[1] "Part 11: Wireless LAN medium access control (MAC) and physical layer (PHY) specification," IEEE, August 1999.

[2] "Medium access control (MAC) enhancements for quality of service (QoS)," IEEE, July 2005.

[3] I. Politis, M. Tsagkaropoulos, S. Kotsopoulos, T. Dagiuklas, and P. Stavroulakis, "On the QoS assesessment of video sessions in heterogeneous 3G-WLANS networks with seamless and secure mobility support," Journal of China Communications, vol. 4, no. 1, 2007, special issue on Communications and Information Security.

[4] G. Bianchi, "Performance analysis of the IEEE 802.11 distributed coordination function," IEEE Journal on Selected Areas in Communications, vol. 18, no. 3, pp. 535-547, 2000.

[5] C. H. Foh and J. W. Tantra, "Comments on IEEE 802.11 saturation throughput analysis with freezing of backoff counters," IEEE Communications Letters, vol. 9, no. 2, pp. 130-132, 2005.

[6] Y. Xiao, "Performance analysis of IEEE 802.11e EDCF under saturation condition," in Proceedings of the IEEE International Conference on Communications (ICC '04), vol. 1, pp. 170-174, Paris, France, June 2004.

[7] P. E. Engelstad and O. N. Østerbø, "Delay and throughput analysis of IEEE 802.11e EDCA with starvation prediction," in Proceedings of the 30th Anniversary IEEE Conference on Local Computer Networks (LCN '05), pp. 647-655, Sydney, Australia, November 2005.

[8] G. S. Paschos, I. Papapanagiotou, S. A. Kotsopoulos, and G. K. Karagiannidis, "A new MAC protocol with pseudo-TDMA behavior for supporting quality of service in 802.11 wireless LANs," EURASIP Journal on Wireless Communications and Networking, vol. 2006, Article ID 65836, 9 pages, 2006.

[9] Y. C. Tay and K. C. Chua, "A capacity analysis for the IEEE 802.11 MAC protocol," Wireless Networks, vol. 7, no. 2, pp. 159-171, 2001.

[10] G. Bianchi and I. Tinnirello, "Remarks on IEEE 802.11 DCF performance analysis," IEEE Communications Letters, vol. 9, no. 8, pp. 765-767, 2005.

[11] J. Choi, J. Yoo, and C.-K. Kim, "A novel performance analysis model for an IEEE 802.11 wireless LAN," IEEE Communications Letters, vol. 10, no. 5, pp. 335-337, 2006.

[12] J. Hui and M. Devetsikiotis, "A unified model for the performance analysis of IEEE 802.11e EDCA," IEEE Transactions on Communications, vol. 53, no. 9, pp. 1498-1510, 2005.

[13] Q. Ni, T. Li, T. Turletti, and Y. Xiao, "Saturation throughput analysis of error-prone 802.11 wireless networks," Wireless Communications and Mobile Computing, vol. 5, no. 8, pp. 945-956, 2005.

[14] T. Li, Q. Ni, T. Turletti, and Y. Xiao, "Performance analysis of the IEEE 802.11e block ACK scheme in a noisy channel," in Proceedings of the 2nd International Conference on Broadband Networks (BROADNETS '05), vol. 1, pp. 551-557, Boston, Mass, USA, October 2005. 
[15] A. Banchs and L. Vollero, "A delay model for IEEE 802.11e EDCA," IEEE Communications Letters, vol. 9, no. 6, pp. 508510, 2005.

[16] J. Abate and W. Whitt, "Numerical inversion of probability generating functions," Operations Research Letters, vol. 12, no. 4, pp. 245-251, 1992. 

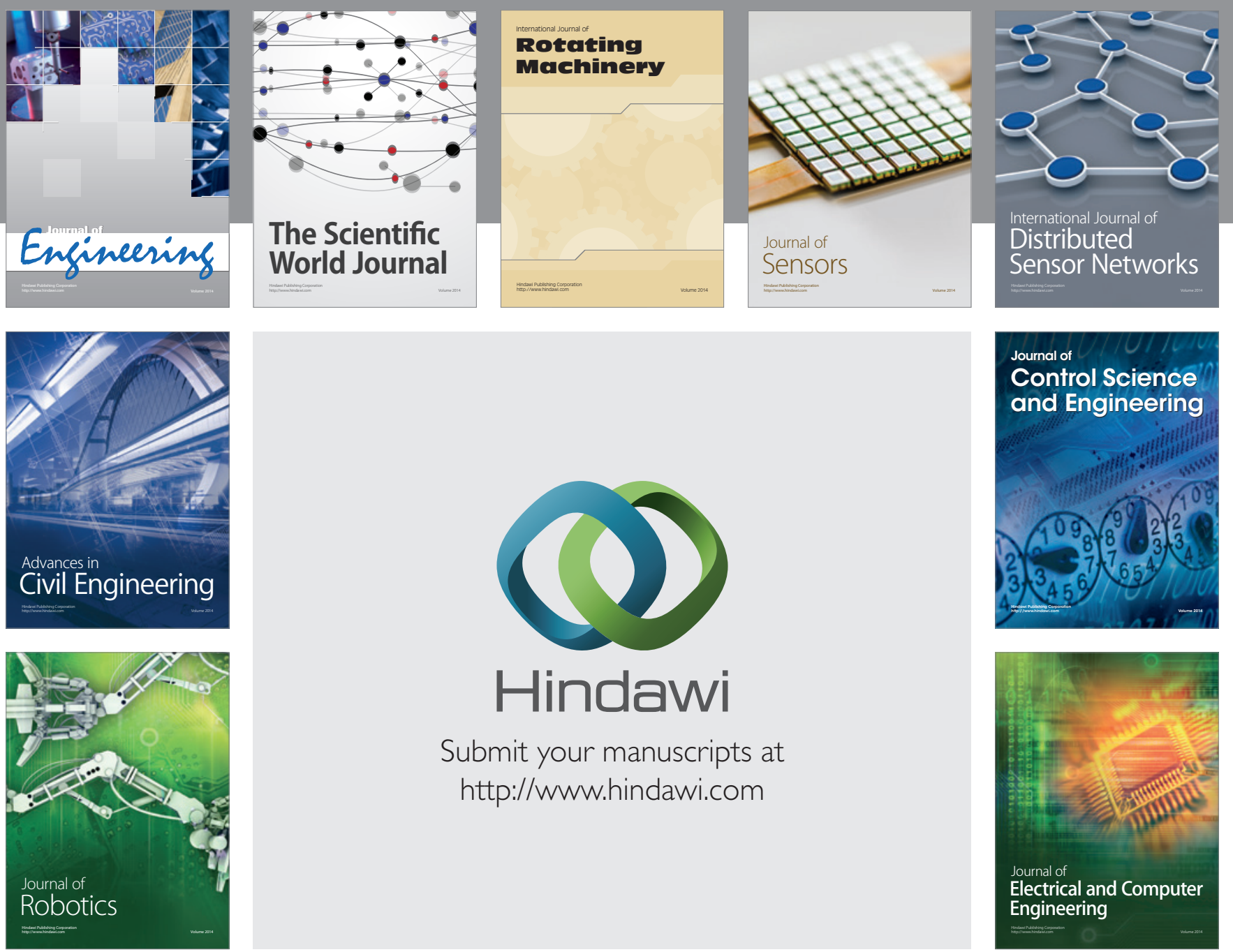

Submit your manuscripts at

http://www.hindawi.com
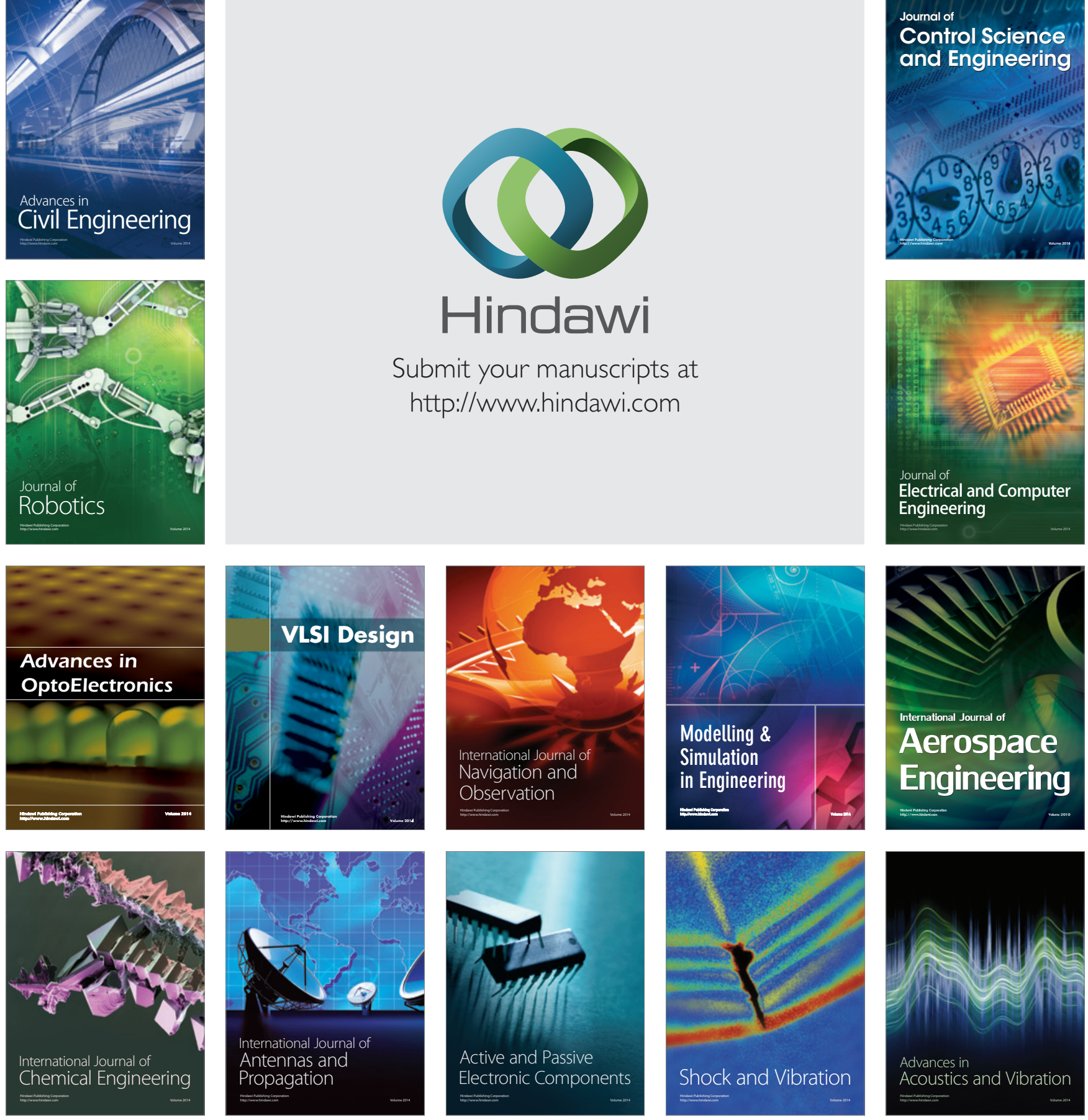